\title{
Urethra-sparing surgery for a prostate cancer lesion in the anterior urethral zone with magnetic resonance-guided focused ultrasound: a case report
}

\author{
Miao Wang, ${ }^{1,2 \#}$, Lei Zhang ${ }^{1 \#}$, Huimin Hou ${ }^{1}$, Tao Gu ${ }^{3}$, Cheng Shen ${ }^{3}$, Xin Ding ${ }^{1}$, Jintao Zhang ${ }^{3}$, \\ Xuan Wang ${ }^{1}$, Jianlong Wang ${ }^{1}$, Jianye Wang ${ }^{1}$, Ming Liu ${ }^{1}$ \\ ${ }^{1}$ Department of Urology, Beijing Hospital, National Center of Gerontology, Institute of Geriatric Medicine, Chinese Academy of Medical Sciences, \\ Beijing, China; ${ }^{2}$ Graduate School of Peking Union Medical College, Chinese Academy of Medical Sciences, Beijing, China; ${ }^{3}$ Department of \\ Radiology, Beijing Hospital, National Center of Gerontology, Institute of Geriatric Medicine, Chinese Academy of Medical Sciences, Beijing, China \\ \#These authors contributed equally and should be considered co-first authors. \\ Correspondence to: Ming Liu. No. 1 Dongdan Dahua Road, Dongcheng District, Beijing 100730, China. Email: liumingbjh@126.com.
}

\begin{abstract}
Prostate cancer $(\mathrm{PCa})$ is the most common malignancy among men worldwide. High-intensity focused ultrasound (HIFU) is a focal therapeutic strategy for low- to intermediate-risk PCa with a low risk of complications. However, lesions located in the anterior urethral zone are challenging to treat with this approach because it is difficult to avoid urethral injury during HIFU therapy, which might further cause urethral stricture and symptoms related to bladder outlet obstruction (BOO). Here, we present the case of a 79-year-old male with elevated prostate-specific antigen (PSA) levels for over 1 year. Multiparametric magnetic resonance imaging revealed a $1.8 \mathrm{~cm} \times 1.0 \mathrm{~cm}$ mass covering an area of the anterior urethral zone. Histopathological examination revealed an International Society of Urological Pathology grade group 3 acinar adenocarcinoma. Given the patient's request for functional preservation, a magnetic resonance-guided focused ultrasound surgery was performed. During the operation, a urethra-sparing approach was utilized by administering proper energy and adjusting the sequence of the sonications. The patient developed urinary retention after catheter removal less than $48 \mathrm{~h}$ after the treatment, which resolved after removal of the second catheter a week later. On a follow-up visit 3 months after the treatment, evaluation by the level of PSA and multiparametric magnetic resonance imaging showed no recurrence of PCa. No significant changes in the International Prostate Symptom Score and Quality of Life score were found compared to baseline scores. With proper adjustment, magnetic resonance-guided focused ultrasound surgery could be safely used for urethra-sparing surgeries for PCa lesions in the anterior urethral zone, without influencing posttreatment urination. The indwelling time of the catheter should be extended appropriately for full recovery from treatment-related prostatic edema around the prostatic urethra to avoid urinary retention.
\end{abstract}

Keywords: Prostate neoplasm; focal therapy; magnetic resonance guided surgery; urethra-sparing; functional preservation; case report

Submitted Aug 27, 2021. Accepted for publication Nov 21, 2021.

doi: $10.21037 /$ tcr-21-1757

View this article at: https://dx.doi.org/10.21037/tcr-21-1757

\section{Introduction}

Prostate cancer $(\mathrm{PCa})$ is the most common malignancy among men worldwide (1). High-intensity focused ultrasound (HIFU) surgery is a focal therapeutic strategy for low- to intermediate-risk PCa with a low incidence of treatment-related complications compared to traditional whole-gland therapies such as radical prostatectomy (RP) and radiotherapy (RT) (2), which has also been proven to have acceptable survival and oncological outcomes with over 5-year follow-up data (3-6). Previously reported HIFU 

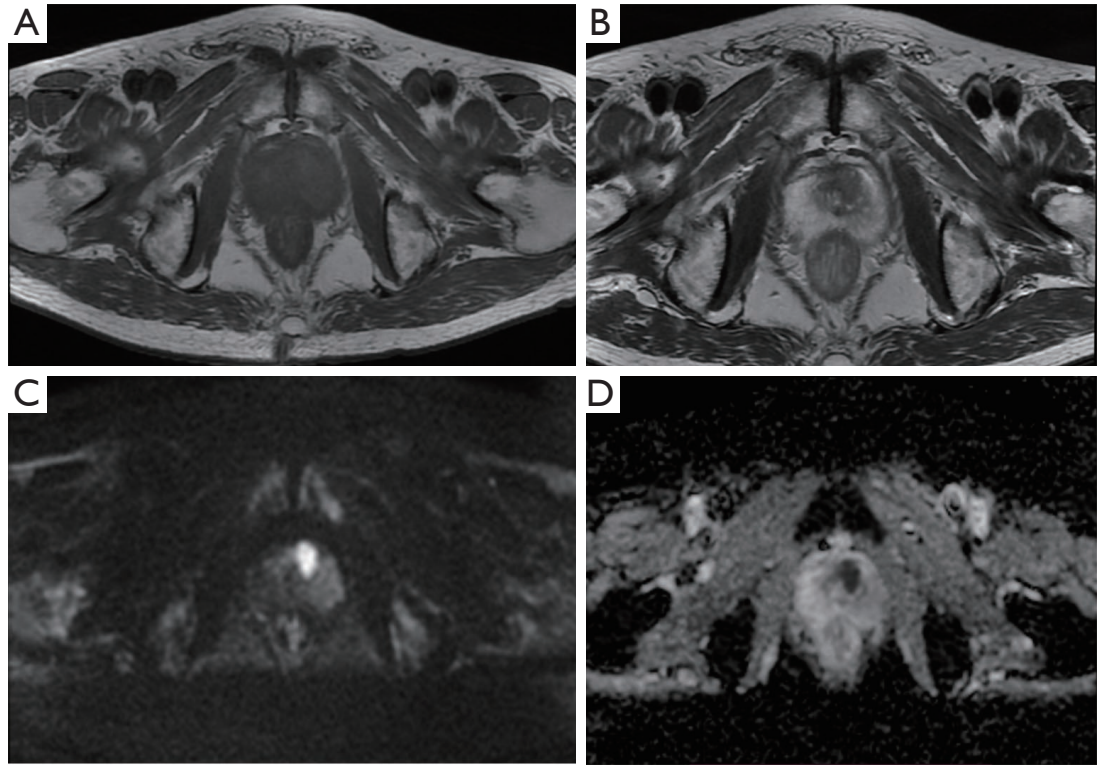

Figure 1 Pre-MRgFUS pelvic mp-MRI. (A) T1WI; (B) an inverted L-shaped lesion with a low signal was found on T2WI, covering an area in the anterior urethral zone; (C,D) A lesion within the left transitional zone with a high signal on DWI (C) and low signal on ADC map (D).

surgery generally used a hemi-gland or extended hemigland ablation scheme $(2,7)$, which increases susceptibility to urethral injury. As a result, post-treatment bladder outlet obstruction (BOO) remains a common complication, the incidence of which could reach as high as $41 \%(8,9)$. Shoji et al. reported a urethra-sparing HIFU strategy (10); however, PCa lesions located in the anterior urethral zone are challenging to treat using this approach. Though focal HIFU is a promising method in overcoming the above challenge, it has been proven in previous study that patients with lesions in the anterior portion of the transitional zone treated with focal HIFU has a higher risk of urinary dysfunction 1 month after the treatment (11). Magnetic resonance-guided focused ultrasound surgery (MRgFUS) could achieve complete focal therapy with more accurate lesion location and real-time monitoring by multiparametric magnetic resonance imaging (mp-MRI) $(12,13)$. Here, we report the case of an elderly male with PCa covering an area of the anterior urethral zone, and a feasible technique for urethra-sparing surgery using $\mathrm{MRgFUS}$. We present the following article in accordance with CARE Guidelines (14) and the CARE reporting checklist (available at https:// dx.doi.org/10.21037/tcr-21-1757).

\section{Case presentation}

A 79-year-old male presented with an elevated prostate- specific antigen (PSA) level of $8.766 \mathrm{ng} / \mathrm{mL}$ without any symptoms. The medical history of the patient included hypertension, nephron-sparing surgery, and radical left hemicolectomy. Digital rectal examination revealed no palpable nodules.

Urine analysis showed no signs of urinary infection. The results of pelvic mp-MRI revealed a mass approximately $1.8 \mathrm{~cm} \times 1.0 \mathrm{~cm}$ in dimension, with a low signal on $\mathrm{T} 2-$ weighted imaging (T2WI) and a high signal on diffusion weighted imaging (DWI) in the transitional zone, covering an area of the anterior urethral zone at 12 o'clock on T2WI. Part of the mp-MRI images are shown in Figure 1. No metastasis was found on prostate-specific membrane antigen positron emission tomography/computed tomography (PSMA PET/CT). A cognitive fusion transperineal target plus systematic mapping biopsy was performed. The results of histopathology demonstrated an International Society of Urological Pathology (ISUP) grade group 3 prostatic acinar adenocarcinoma, with the location of the lesion consistent with that on mp-MRI.

After fully informing the patient about the condition and treatment options, the patient chose to receive MRgFUS treatment in consideration of less trauma and functional preservation. A suprapubic catheter rather than a urethral catheter was inserted before the procedure to avoid an impact on the transmission of energy. The procedure was performed using an endorectal focused ultrasound ablation system 

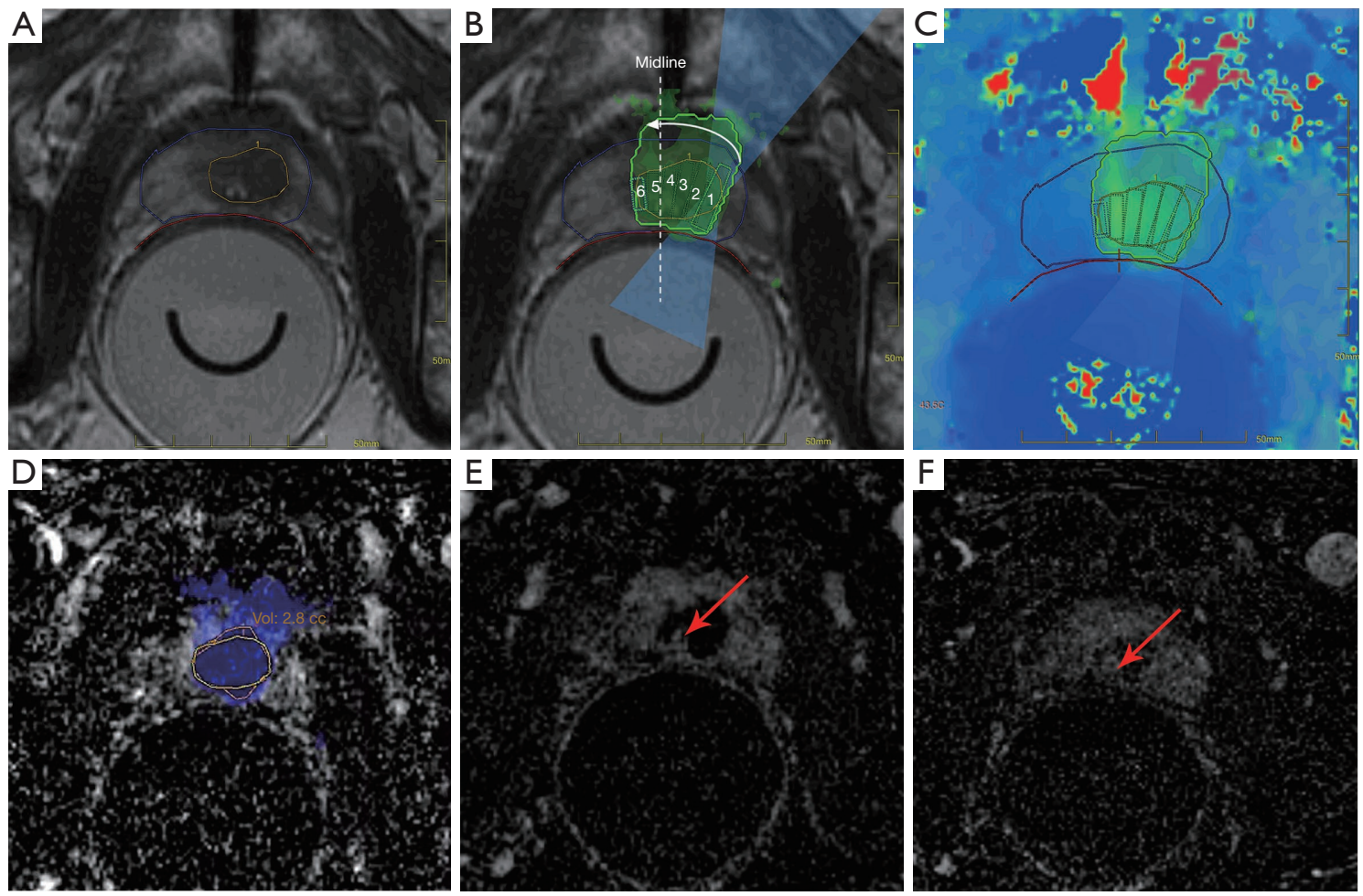

Figure 2 MRI during MRgFUS surgery and immediate post-MRgFUS MRI. (A) Pretreatment MRI shows the contoured rectal wall (red outline), prostate capsule (blue outline), and the region of treatment with a 5-mm tumor free margin (yellow outline) (B) MRI during the treatment process shows the macro spot planned for the target lesion (green rectangle) and the beam path (blue). The arrows and numbers show the direction and sequence of sonication, respectively; (C) The thermal map during the treatment shows heat deposition. Red is for high temperature (in this case, most red areas are due to artifacts, because temperatures in bones and the transducer could not be measured), green for thermal dose deposition, and blue is for low temperature; (D) Axial T1 contrast-enhanced image shows the non-perfused volume (orange outline) overlaid on the region of treatment by plan. (E,F) Post-treatment contrast-enhanced MRI shows enhanced urethral mucosa (red arrow) on the adjacent layer of sonication.

(ExAblate 2100, InSightec, Haifa, Israel) integrated within a 3T MR scanner (Discovery MR750, GE Medical Systems, Milwaukee, Wisconsin, USA). The patient was positioned supine on the patient table under general anesthesia. After insertion of the probe and localization, the prostatic capsule, anterior surface of the rectal wall, and region of treatment (ROT) with at least 5 -mm tumor-free margins were manually contoured on axial T2WI (Figure 2A). A total of 3 layers were planned for the treatment, with a $3 \mathrm{~mm}$-interval between layers. In order to treat the lesion completely, we did not delineate the actual outline of the lesion, which appeared as an "inverted L shape" on T2WI imaging (Figure 1B); instead, an elliptic area was delineated covering both the urethra and the complete lesion with a safety margin of over $5 \mathrm{~mm}$ (Figure $2 A$ ). As the urethra was within the ROT, we planned to minimize injury to the urethra. Specifically, we rearranged the sequence of sonication of the macro spots, as shown by the green rectangle in Figure 2B. In order to minimize the thermal damage to the urethra (i.e., due to direct delivery of energy through or near the urethra), each sonication started from the lateral edge of the prostate instead of starting from the medial part of the prostate, as shown in Figure 2B. Thus, the accumulated thermal dose absorbed by the urethra, which was closest to the lesion located at the midline of the prostate, was reduced to a minimum. During the entire treatment, real-time temperature monitoring was realized by an overlay of MRI thermography over the anatomic images (Figure 2C), and a temperature over $65{ }^{\circ} \mathrm{C}$ within the target lesion was considered adequate for the treatment. After completion of sonication, a contrast-enhanced MRI was performed, which showed a non-perfused volume (NPV, 

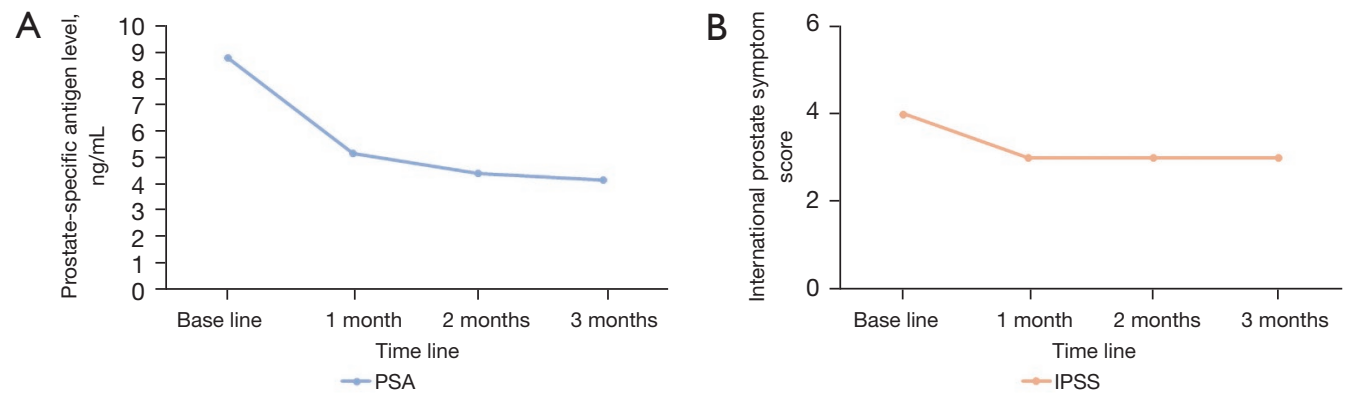

Figure 3 PSA and IPSS trend after MRgFUS surgery. (A) PSA at baseline, 1st, 2nd, and 3rd month after the treatment, respectively; (B) IPSS at baseline, 1st, 2nd, and 3rd month after the treatment, respectively.

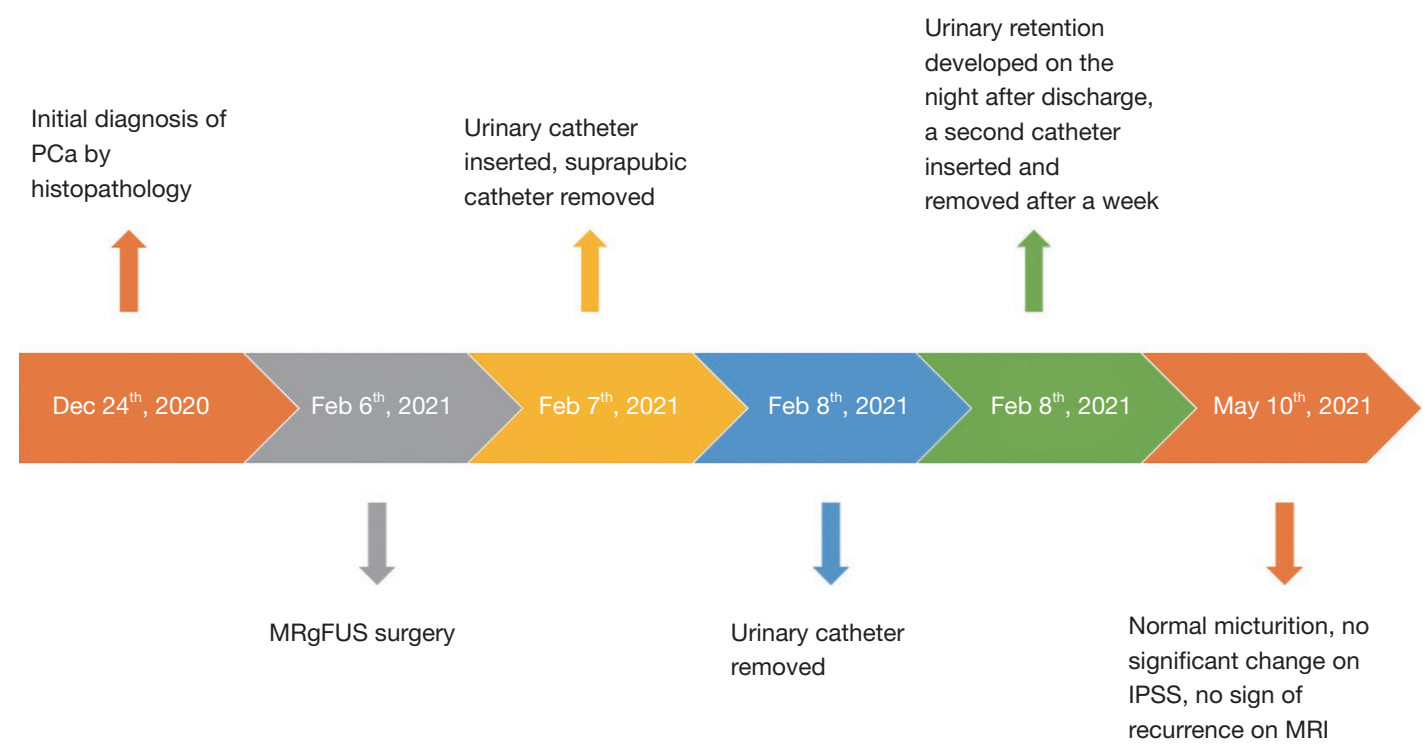

Figure 4 Timeline of management.

$2.8 \mathrm{cc})$ covering the ROT outlined preoperatively (Figure 2D). An approximately inverted L-shaped NPV was observed on the adjacent layer on enhanced MRI, with the urethra enhanced on the imaging, suggesting preserved tissue activity (Figure 2E,2F).

After the treatment, the suprapubic catheter was replaced by a urethral catheter, which was removed after a day, before discharge from the hospital. The patient recovered spontaneous micturition immediately after catheter removal but developed urinary retention on the night of discharge. A 16F Foley catheter was re-inserted and indwelled for 1 week before removal. Thereafter, the recovery was uneventful. The PSA level and International Prostate Symptom Score (IPSS) and Quality of Life (QoL) score were monitored at 1,2 , and 3 months after the treatment, the trends of which are shown in Figure 3, with a significant decline in the PSA level but no obvious changes in IPSS compared to the baseline score. The QoL score before MRgFUS and 1-, 2 - and 3-month after the treatment were all 0 according to the questionaries acquired from the patient. The result of mp-MRI at the 3-month follow-up visit showed no signs of recurrence. The timeline of the medical history and management of the patient is shown in Figure 4.

The patient presented in this case was from a prospectively designed study (ChiCTR2000034634) approved by the Institutional Review Board of Beijing Hospital. All procedures performed in studies involving human participants were in accordance with the ethical standards of the institutional and/or national research committee(s) and with the Helsinki Declaration (as revised 
in 2013). Written informed consent was obtained from the patient for publication of this case report and accompanying images. A copy of the written consent is available for review by the editorial office of this journal.

\section{Discussion}

The appropriate management of $\mathrm{PCa}$ is of paramount importance, considering its high prevalence among men. The traditional approach for low- to intermediaterisk $\mathrm{PCa}$ is mainly whole-gland therapy, including $\mathrm{RP}$ and RT (15), which is associated with a considerable incidence of complications such as erectile dysfunction, urinary incontinence, and rectitis, significantly impacting patients' quality of life (QoL). Active surveillance (AS) is an alternative to avoid overtreatment and treatment-related complications; however, it adds to the patient's psychological and financial burden. Moreover, patients under AS have a chance to miss the opportunity for a cure, especially those with intermediate-risk PCa (16). HIFU therapy is a novel focal therapeutic strategy for $\mathrm{PCa}$, representing the middle ground between whole-gland therapy and AS. HIFU uses the energy of ultrasound waves to destroy cancer cells by initiating the process of coagulative necrosis (17). Treating cancer lesions precisely but preserving normal tissue and function (18), it can reduce the risk of treatment-related complications, greatly improve patients' QoL, and decrease the burden of complication management $(3,4)$.

This case involved an elderly male diagnosed with intermediate-risk PCa in good health condition. Considering the complex history of abdominal and pelvic surgery, aspiration for a cure, and requirement of functional preservation, mainly urinary function, we regarded focal therapy using focused ultrasound as an optimal option. Traditional HIFU mostly uses a hemi-gland or extended hemi-gland ablation scheme to achieve complete tumor ablation due to the difficulty in localization and margin identification by ultrasound (2). Post-treatment BOO remains a common complication, which can be classified as early or delayed types $(8,9)$, resulting from transient edema of the prostatic stroma $(8,9)$ and urethral stricture with fibrosis, respectively (10). Shoji et al. reported a urethrasparing HIFU approach to reduce treatment-related BOO, regarded safe for selected patients and effective for prevention of urethral stricture and delayed-type BOO (10). However, this approach was not appropriate for cancer lesions located in the anterior urethral zone. Moreover, it has also been proven that patients with lesions in the anterior portion of the transitional zone has a higher risk of urinary dysfunction 1 month after the treatment, even though treated by focal HIFU (11).

Taking advantage of the high resolution of soft tissues and real-time temperature monitoring from MRI, the MRgFUS represents a newer technique based on focused ultrasound, which can realize more accurate cancer localization and a closed-loop feedback control of energy deposition, and eventually better fulfill the goal of "focal" ablation. In this case, a mass within the left transitional zone was found, with a high signal on DWI and low signal on the ADC map. However, a comparatively larger lesion was seen on T2WI, with an area involving the anterior urethral zone. As the treatment was planned based on T2WI, a scheme for treating the complete "inverted L-shaped" lesion was established to avoid the risk of incomplete treatment. Injury to the urethra could not be completely avoided as it was situated within the path of energy propagation. Orihuela et al. proved that the prostatic urethra would develop a new epithelial lining over time after thermal injury (19), suggesting that treatment covering the urethra is a safe procedure. However, urethral stricture with fibrosis resulting in $\mathrm{BOO}$ was also reported after $\mathrm{HIFU}$, probably due to the relatively extended treatment area. Thus, we hypothesized that by reducing the injury to the urethra and adjacent normal tissue, the risk of developing earlyand delayed-type BOO could be reduced to a minimum. As the exposure temperature and exposure time under the temperature are two key parameters associated with cell necrosis (20), we fulfilled our goal by adjusting the sequence of sonication of the macro spots, starting from the lateral side, the spots at which were the farthest from the urethra, to the midline of the prostate. In this way, the first subspots of the sonication, which are usually the ones with the highest energy in comparison to the rest of the subspots in the macro-sonication, were located far away from the urethra. In turn, the spots on/near the urethra would be with less energy, mainly thanks to the accumulated heat from previous spots, which allowed lower energy to reach the required thermal dose. Using this approach, we reduced the persistent exposure time of the midline tissue, located closest to the urethra, at a high temperature, thus reducing the accumulated thermal dose. Post-treatment contrastenhanced MRI showed the urethra on the adjacent layer with enhanced signal, demonstrating the efficacy of our method in the preservation of urethral tissue activity. The satisfactory early outcomes also demonstrated that with appropriate adjustments, the treatment with MRgFUS could 
fulfill the goal of avoiding the early obstruction symptoms caused by prostatic swelling. As for the impact on longterm outcomes, follow-up data are still needed; however, since the patient had no sign of dysuria postoperatively, we believed the treatment strategy we proposed here a safe method for a urethra-sparing surgery.

There is no consensus regarding the time for catheter removal after $\mathrm{MRgFUS}$ treatment. A range of 0-7 days after treatment before catheter removal was reported by previous studies $(12,21,22)$. In this case, a suprapubic catheter was used first as the urethra lay in the path of energy propagation. It was replaced by a urethral catheter, which was removed at less than $48 \mathrm{~h}$ after the treatment. However, urinary retention occurred, necessitating re-insertion of the catheter, which was removed 7 days later. The cause of retention was mostly attributed to edema of the prostatic stroma. Specifically, edema might have occurred very close to the urethra in this case, causing obstruction. Therefore, for patients with $\mathrm{PCa}$ lesions adjacent to the urethra or with the urethra within the treatment area of MRgFUS, a prolonged catheter-indwelling time may be necessary.

Previous studies reported some preliminary results proving MRgFUS as a feasible and safe approach for PCa treatment, with acceptable oncological outcomes and satisfactory functional outcomes $(12,13,21,23)$ and complication rates far below that of non-MR guided focal HIFU. In this case, no obvious unpleasant side effects were reported by the patient. No sign of recurrence was found at the 3-month follow-up visit, and there was no significant change in IPSS, signifying our plan of a urethra-sparing MRgFUS to be safe and feasible.

For patients with low- to intermediate-risk localized $\mathrm{PCa}$, the safety and efficiency of the therapeutic regimen and the impact on QoL should be taken into consideration when making decisions. MRgFUS has the potential to be an alternative to whole-gland therapy with acceptable oncological outcomes and a far lower complication rate. For PCa lesions located in the anterior urethral zone, we proved in this case that MRgFUS can safely be administered with appropriate adjustment during the treatment process. However, the oncological and functional outcomes of our proposed treatment strategy still need to be tested in further studies with larger sample sizes.

The treatment approach using MRgFUS utilized in this case could be safely used for a urethra-sparing surgery of PCa lesions in the anterior urethral zone, without influencing post-treatment urination. The indwelling time of the catheter should be extended appropriately for full recovery from treatment-related prostatic edema around the prostatic urethra to avoid early urinary retention.

\section{Acknowledgments}

Funding: This work was supported by the Fundamental Research Funds for the Central Universities (grant number 3332019122), the Fundamental Research Funds for the Central Universities (grant number 3332020069), and Innovation Funds for Postgraduates of Peking Union Medical College (grant number 2019-1002-87).

\section{Footnote}

Reporting Checklist: The authors have completed the CARE reporting checklist. Available at https://dx.doi. org/10.21037/tcr-21-1757

Peer Review File: Available at https://dx.doi.org/10.21037/ tcr-21-1757

Conflicts of Interest: All authors have completed the ICMJE uniform disclosure form (available at https://dx.doi. org/10.21037/tcr-21-1757). The authors have no conflicts of interest to declare.

Ethical Statement: The authors are accountable for all aspects of the work in ensuring that questions related to the accuracy or integrity of any part of the work are appropriately investigated and resolved. All procedures performed in studies involving human participants were in accordance with the ethical standards of the institutional and/or national research committee(s) and with the Helsinki Declaration (as revised in 2013). Written informed consent was obtained from the patient for publication of this case report and accompanying images. A copy of the written consent is available for review by the editorial office of this journal.

Open Access Statement: This is an Open Access article distributed in accordance with the Creative Commons Attribution-NonCommercial-NoDerivs 4.0 International License (CC BY-NC-ND 4.0), which permits the noncommercial replication and distribution of the article with the strict proviso that no changes or edits are made and the original work is properly cited (including links to both the formal publication through the relevant DOI and the license). See: https://creativecommons.org/licenses/by-nc-nd/4.0/. 


\section{References}

1. Miller KD, Nogueira L, Mariotto AB, et al. Cancer treatment and survivorship statistics, 2019. CA Cancer J Clin 2019;69:363-85.

2. Perera M, Krishnananthan N, Lindner U, et al. An update on focal therapy for prostate cancer. Nat Rev Urol 2016;13:641-53.

3. Guillaumier S, Peters M, Arya M, et al. A Multicentre Study of 5-year Outcomes Following Focal Therapy in Treating Clinically Significant Nonmetastatic Prostate Cancer. Eur Urol 2018;74:422-9.

4. Stabile A, Orczyk C, Hosking-Jervis F, et al. Medium-term oncological outcomes in a large cohort of men treated with either focal or hemi-ablation using high-intensity focused ultrasonography for primary localized prostate cancer. BJU Int 2019;124:431-40.

5. Lovegrove CE, Peters M, Guillaumier S, et al. Evaluation of functional outcomes after a second focal high-intensity focused ultrasonography (HIFU) procedure in men with primary localized, non-metastatic prostate cancer: results from the HIFU Evaluation and Assessment of Treatment (HEAT) registry. BJU Int 2020;125:853-60.

6. Tourinho-Barbosa RR, Sanchez-Salas R, Claros OR, et al. Focal Therapy for Localized Prostate Cancer with Either High Intensity Focused Ultrasound or Cryoablation: A Single Institution Experience. J Urol 2020;203:320-30.

7. Lindner U, Trachtenberg J, Lawrentschuk N. Focal therapy in prostate cancer: modalities, findings and future considerations. Nat Rev Urol 2010;7:562-71.

8. Blana A, Hierl J, Rogenhofer S, et al. Factors predicting for formation of bladder outlet obstruction after highintensity focused ultrasound in treatment of localized prostate cancer. Urology 2008;71:863-7.

9. Netsch C, Pfeiffer D, Gross AJ. Development of bladder outlet obstruction after a single treatment of prostate cancer with high-intensity focused ultrasound: experience with 226 patients. J Endourol 2010;24:1399-403.

10. Shoji S, Nakano M, Fujikawa H, et al. Urethra-sparing high-intensity focused ultrasound for localized prostate cancer: Functional and oncological outcomes. Int J Urol 2015;22:1043-9.

11. Hanada I, Shoji S, Takeda K, et al. Significant Impact of the Anterior Transition Zone Portion Treatment on Urinary Function After Focal Therapy with HighIntensity Focused Ultrasound for Prostate Cancer. J Endourol 2021;35:951-60.

12. Ghai S, Louis AS, Van Vliet M, et al. Real-Time MRI-
Guided Focused Ultrasound for Focal Therapy of Locally Confined Low-Risk Prostate Cancer: Feasibility and Preliminary Outcomes. AJR Am J Roentgenol 2015;205:W177-84.

13. Tay KJ, Cheng CWS, Lau WKO, et al. Focal Therapy for Prostate Cancer with In-Bore MR-guided Focused Ultrasound: Two-Year Follow-up of a Phase I Trial-Complications and Functional Outcomes. Radiology 2017;285:620-8.

14. Riley DS, Barber MS, Kienle GS, et al. CARE guidelines for case reports: explanation and elaboration document. J Clin Epidemiol 2017;89:218-35.

15. Mottet N, van den Bergh RCN, Briers E, et al. EAUEANM-ESTRO-ESUR-SIOG Guidelines on Prostate Cancer-2020 Update. Part 1: Screening, Diagnosis, and Local Treatment with Curative Intent. Eur Urol 2021;79:243-62.

16. Klotz L, Vesprini D, Sethukavalan P, et al. Long-term follow-up of a large active surveillance cohort of patients with prostate cancer. J Clin Oncol 2015;33:272-7.

17. Chapelon JY, Margonari J, Vernier F, et al. In vivo effects of high-intensity ultrasound on prostatic adenocarcinoma Dunning R3327. Cancer Res 1992;52:6353-7.

18. Donaldson IA, Alonzi R, Barratt D, et al. Focal therapy: patients, interventions, and outcomes--a report from a consensus meeting. Eur Urol 2015;67:771-7.

19. Orihuela E, Pow-Sang M, Motamedi M, et al. Mechanism of healing of the human prostatic urethra following thermal injury. Urology 1996;48:600-8.

20. Sapareto SA, Dewey WC. Thermal dose determination in cancer therapy. Int J Radiat Oncol Biol Phys 1984;10:787-800.

21. Napoli A, Anzidei M, De Nunzio C, et al. Real-time magnetic resonance-guided high-intensity focused ultrasound focal therapy for localised prostate cancer: preliminary experience. Eur Urol 2013;63:395-8.

22. Lindner U, Ghai S, Spensieri P, et al. Focal magnetic resonance guided focused ultrasound for prostate cancer: Initial North American experience. Can Urol Assoc J 2012;6:E283-6.

23. Ghai S, Perlis N, Lindner U, et al. Magnetic resonance guided focused high frequency ultrasound ablation for focal therapy in prostate cancer - phase 1 trial. Eur Radiol 2018;28:4281-7.

Cite this article as: Wang $M$, Zhang L, Hou H, Gu T, Shen C, Ding X, Zhang J, Wang X, Wang J, Wang J, Liu M. Urethra-sparing surgery for a prostate cancer lesion in the anterior urethral zone with magnetic resonance-guided focused ultrasound: a case report. Transl Cancer Res 2021;10(12) 5430-5436. doi: 10.21037/tcr-21-1757 